\title{
Superdiffusion, large-scale synchronization and topological defects
}

\author{
Robert Großmann, ${ }^{1, \text { f F Fernando Peruani, }}{ }^{2}$ and Markus Bär ${ }^{1}$ \\ ${ }^{1}$ Physikalisch-Technische Bundesanstalt Berlin, Abbestr. 2-12, 10587 Berlin, Germany \\ ${ }^{2}$ Laboratoire J. A. Dieudonné, Université de Nice Sophia Antipolis, \\ UMR 7351 CNRS, Parc Valrose, F-06108 Nice Cedex 02, France
}

(Dated: January 23, 2020)

\begin{abstract}
We study an ensemble of random walkers carrying internal noisy phase oscillators which are synchronized among the walkers by local interactions. Due to individual mobility, the interaction partners of every walker change randomly, hereby introducing an additional, independent source of fluctuations, thus constituting the intrinsic nonequilibrium nature of the temporal dynamics. We employ this paradigmatic model system to discuss how the emergence of order is affected by motion of individual entities. In particular, we consider both, normal diffusive motion and superdiffusion. A non-Hamiltonian field theory including multiplicative noise terms is derived which describes the nonequilibrium dynamics at the macroscale. This theory reveals a defect-mediated transition from incoherence to quasi long-range order for normal diffusion of oscillators in two dimensions, implying a power-law dependence of all synchronization properties on system size. In contrast, superdiffusive transport suppresses the emergence of topological defects, thereby inducing a continuous synchronization transition to long-range order in two dimensions. These results are consistent with particle-based simulations.
\end{abstract}

PACS numbers: 05.45.Xt, 05.10.Gg, 05.40.Fb, 64.60.De

\section{INTRODUCTION}

In a large variety of chemical and biological systems, moving entities synchronize an internal degree of freedom: examples include mobile catalytic beads exhibiting the oscillatory Belousov-Zhabotinsky (BZ) reaction [1] as well as the BZ reaction in vortical flow 2, synthetic genetic oscillators [3, motile cells synchronizing intracellular oscillations during embryonic somitogenesis [4 6], quorum sensing of signaling amoebae [7] and the coordinated motion of myxobacteria regulated by the oscillatory Frz-signaling system 8 10. Furthermore, it has been speculated that the flagellar beating of active microswimmers synchronizes by hydrodynamic interaction thus affecting the rheology of active suspensions [11, 12].

Motivated by the above-mentioned nonequibrium systems, we study synchronization in an ensemble of either diffusively or superdiffusively moving random walkers carrying internal noisy oscillators which synchronize by local interactions. This paradigmatic model describes a nonequilibrium system as evidenced by an analogy to Monte-Carlo algorithms [13: random motion corresponds to Kawasaki dynamics [14 at infinite temperature, whereas the oscillator dynamics in the presence of noise is similar to Glauber updates [15] at a finite temperature. Ergo, the system is coupled to two heat baths simultaneously and, as a consequence, does never reach thermodynamic equilibrium.

We are primarily addressing the question how the emergence of order in spatially extended systems, which relies on the suppression of fluctuations by sufficiently

\footnotetext{
* grossmann@physik.hu-berlin.de
}

fast information transfer through the system, is influenced by the motion type of individual entities. This question is of interest beyond synchronization in nonequilibrium statistical mechanics, ranging from active matter [16 -18 and collective motion [19, 20, to stochastic reaction-diffusion systems [21, 22] and epidemic spreading 23 25]. Ultimately, the interplay of local interaction and transport resulting in the emergence of order is a key element in all the above-named applications.

The study of phase transitions and spontaneous symmetry breaking in isothermal systems at thermodynamic equilibrium has already led to fundamental theoretical insights regarding the emergence of order such as the universality of critical behavior [26] or the Mermin-Wagner theorem [27, which excludes the emergence of long-range order in low-dimensional equilibrium systems with a continuous symmetry at finite temperature. This work focusses on the crucial influence of the motility of individual entities on the emergence of macroscopic order in nonequilibrium systems, which is less well understood.

The temporal dynamics of many oscillators models, such as phase oscillators which constitute one of the corner stones of synchronization theory [28, is invariant under global phase shifts. This leads to the emergence of spin-waves in one dimension [29, 30] and topological defects [31] in two dimensions. These facts, that relate synchronization theory and statistical mechanics, were often overlooked in studies of motile oscillators [32 38. Here, we discuss how the motion of these topological excitations and the corresponding annihilation dynamics is affected by the motion of the oscillators.

Whereas various previous studies focused on deterministic oscillators [32 36, we examine fluctuation-induced properties of synchronized states such as correlation functions and study synchronization from a statistical me- 
chanics perspective. In particular, we systematically derive a field theory from the oscillator model and demonstrate that oscillators moving diffusively exhibit a defectmediated transition from incoherence to quasi long-range order (QLRO) analogous to the Berezinskii-KosterlitzThouless (BKT) transition [39, 40] in two dimensions. We show further that a continuous nonequilibrium transition to long-range order (LRO) does occur - forbidden in equilibrium systems according to the MerminWagner theorem [27] - if oscillators move superdiffusively, thereby accelerating the annihilation of defects.

\section{MODEL}

We consider an ensemble of $N$ random walkers in a periodic space, each of them carrying an internal clock. Individual clocks, which are assumed to possess identical mean natural frequencies $\omega_{0}$, are modeled as noisy phase oscillators that synchronize to the clocks of neighboring walkers. The state of the $j$-th walker is thus characterized by its position $\mathbf{r}_{j}(t)$ and the phase $\theta_{j}(t)$ or the relative phase $\chi_{j}(t)=\theta_{j}(t)-\omega_{0} t$. The dynamics of $\mathbf{r}_{j}(t)$ and $\chi_{j}(t)$ obeys the Langevin equations

$$
\begin{aligned}
\dot{\mathbf{r}}_{j} & =\boldsymbol{\xi}_{j}(t) \\
\dot{\chi}_{j} & =\frac{1}{N_{j}} \sum_{k \neq j} \beta\left(\left|\mathbf{r}_{k}-\mathbf{r}_{j}\right|\right) \sin \left(\chi_{k}-\chi_{j}\right)+\sqrt{2 D_{\chi}} \eta_{j}(t) .
\end{aligned}
$$

This model system can be understood as a nonequilibrium, off-lattice version of the XY model where individual spins are motile.

The random motion of particles (or spins) is described by Eq. (1a), where $\boldsymbol{\xi}_{j}(t)$ denotes Lévy noise [41, 42. Hence, a particle jumps according to $\mathbf{r}_{j}(t+\tau)=$ $\mathbf{r}_{j}(t)+\int_{t}^{t+\tau} d t^{\prime} \boldsymbol{\xi}_{j}\left(t^{\prime}\right)$ in a time interval $\tau>0$. The random displacements follow a Lévy $\alpha$-stable distribution [43] defined by its propagator $G_{\alpha}(\mathbf{r}, \tau)$ in Fourier domain: $\hat{G}_{\alpha}(\mathbf{q}, \tau)=e^{-\gamma \tau|\mathbf{q}|^{\alpha}}$. The motion is characterized by a generalized diffusion coefficient $\gamma$ determining the width of the displacement distribution, and an in$\operatorname{dex} \alpha$ describing the tails of the step length distribution. For $\alpha=2$, the displacement distribution is Gaussian and, consequently, particles undergo Brownian motion with a diffusion coefficient $\gamma$. In contrast, the step length distribution is heavy tailed for $\alpha \in(0,2)$ implying large jumps. Hence, walkers perform Lévy flights [41, 44].

Eq. (1b) describes the oscillator dynamics. The first term accounts for the local interaction among oscillators analogous to the paradigmatic Kuramoto model [28, 4547]. The interaction strength $\beta$ of two oscillators is a function of their relative distance. We assume that the interaction is strictly short-ranged, i.e. it vanishes beyond a certain interaction range representing the sensing radius of an individual oscillator that we set to one: $\beta(x)=0$ for $|x|>1$. The interaction is rescaled by the number of neighbors $N_{j}=\sum_{k \neq j} \beta\left(\left|\mathbf{r}_{k}-\mathbf{r}_{j}\right|\right)$ reflecting that the adaptivity of an oscillator is independent of the number of neighbors. The second term in Eq. $1 \mathrm{~b}$ is an additive, Gaussian white noise [48] with intensity $D_{\chi}$.

\section{PHENOMENOLOGY}

We performed numerical simulations particularly studying the influence of oscillator motion on synchronization in two dimensions. Here, the density of particles is set to $\rho_{0}=1$, which is below the percolation threshold $\rho_{0, \text { perc. }} \approx 1.44$ [50]. Therefore, the communication of oscillators over large scales is dominated by particle transport.

First, we consider the order parameter $\Phi=$ $\left|\sum_{k=1}^{N} e^{i \chi_{k}(t)} / N\right|^{2}$ as a function of the noise strength $D_{\chi}$ (left column in Fig. 1). A transition from incoherence $(\Phi \simeq 0)$ to synchronization $(\Phi>0)$ is observed below a critical noise strength $D_{\chi_{c}}$. The dependence of $D_{\chi_{c}}$ on the diffusivity $\gamma$ indicates that synchronization is enhanced by increasing $\gamma$. For $\gamma \rightarrow \infty$, the order parameter tends to the order parameter for globally coupled oscillators 49. We can conclude that motion enhances information transfer in finite systems, thus promoting the emergence of order.

The transport properties crucially influence the synchronization properties which becomes evident from varying the particle number $N$ and system size $L$ such that the average density $\rho_{0}=N / L^{2}$ is constant (finitesize scaling). For Brownian motion, the order parameter decreases according to a power law with the particle num-
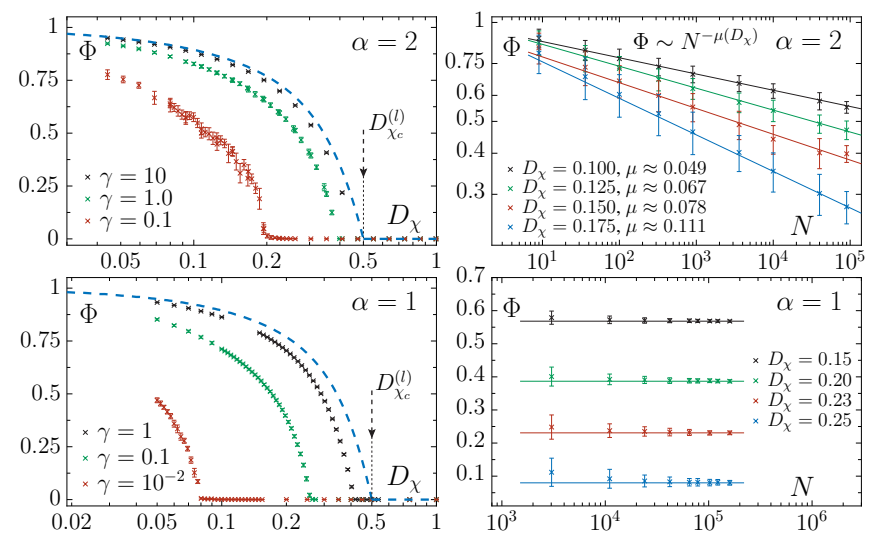

FIG. 1. Order parameter $\Phi$ (averaged over time) as a function of the noise $D_{\chi}$ (left) and the particle number $N$ (right) for oscillators moving diffusively (upper row) and by Lévy flights (lower row). The blue dashed line represents the solution of the Kuramoto model (critical point: $D_{\chi_{c}}^{(l)}=1 / 2$ ) for global coupling [9]. In accordance with the theory (cf. section IV), the lines in the upper right panel correspond to power law fits, while the horizontal lines in the lower right panel indicate the saturation of the order parameter with system size. Parameters: $\rho_{0}=1, \beta(x)=\Theta(1-x), L=300$ (left column), $\gamma=0.1$ (right column), numerical time step $\tau=0.01$. 


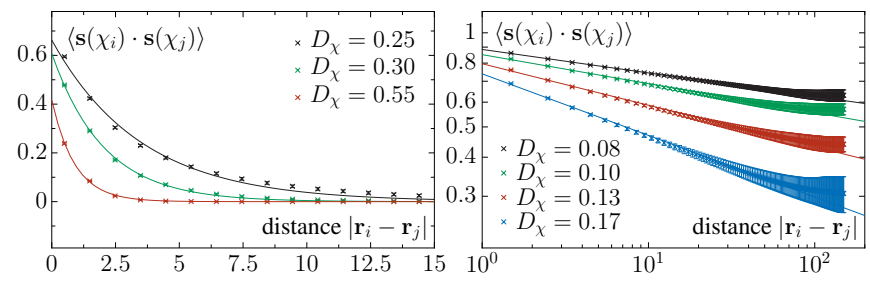

FIG. 2. Correlation function (averaged over time) of $\mathbf{s}\left(\chi_{i}\right)=$ $\left(\cos \chi_{i}, \sin \chi_{i}\right)$ as a function of the distance for diffusing oscillators. For high noise (disordered phase), correlations decay exponentially (left panel). In contrast, correlations decrease algebraically for low noise (right panel) confirming the BKT nature of the synchronization transition and the emergence of QLRO. Lines represent fits using the associated functional form. Parameters: $\alpha=2, \gamma=0.1, \rho_{0}=1, L=300$, $\beta(x)=\Theta(1-x)$, numerical time step $\tau=0.01$.

ber (top right in Fig. 1). The corresponding exponent is a function of the model parameters, particularly the noise strength $D_{\chi}$. Thus, the system is globally disordered in the thermodynamic limit. Similar information is conveyed by the correlation function (Fig. 2). We assigned to each phase variable $\chi_{i}$ a $\operatorname{spin} \mathbf{s}\left(\chi_{i}\right)=\left(\cos \chi_{i}, \sin \chi_{i}\right)$ and calculated the correlations of $\mathbf{s}$ as a function of the distance. Correlations decay exponentially for high noise but decrease according to a power law in the low noise regime as QLRO emerges through a BKT transition [39, 40].

Lévy flights change the qualitative picture significantly: the order parameter $\Phi$ tends towards a nonzero constant value for large system sizes (bottom right in Fig. 1) suggesting the emergence of a robust synchronized state. Further, this result implies, notably, the emergence of long-range order (LRO) in two dimensions. We find that superdiffusion, in contrast to what we observe for diffusive transport, qualitatively affects the large-scale properties of the ordered states and, consequently, the nature of the associated phase transition. In section IV] we confirm these findings by a field theoretic analysis.

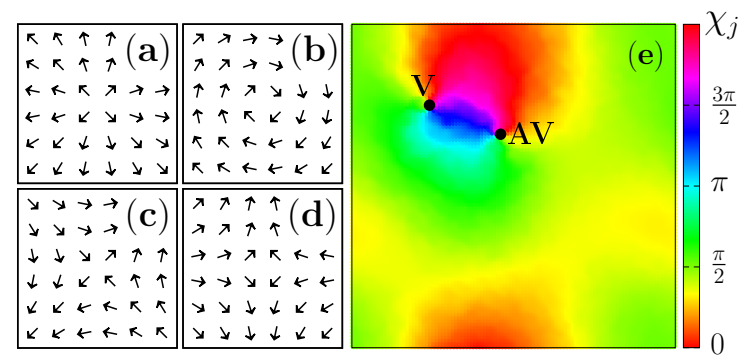

FIG. 3. Relaxational dynamics from random initial conditions: vortices $(\mathrm{V})$ and anti-vortices $(\mathrm{AV})$ are schematically shown by vectors $\mathbf{s}\left(\chi_{j}\right)=\left(\cos \chi_{j}, \sin \chi_{j}\right)$ in panels $(\mathrm{a}, \mathrm{b})$ and $(\mathrm{c}, \mathrm{d})$, respectively. A snapshot of a vortex-anti-vortex pair is depicted in (e), where phases $\chi_{j}$ are color-coded.
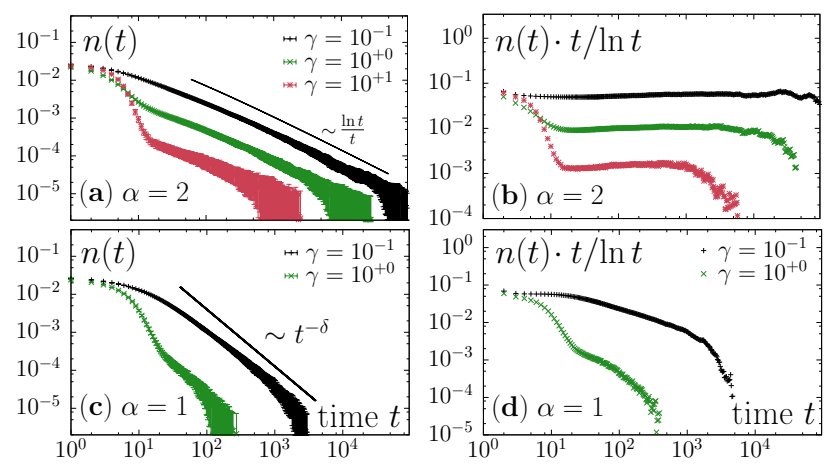

FIG. 4. Relaxational dynamics from random initial conditions: defect density $n(t)$ (averaged over multiple realizations) for Brownian motion (top) and Lévy flights (bottom) as a function of time. On the right, the defect density is rescaled by $t / \ln t$. The black line in (a) also indicates this scaling. Superdiffusive transport (d) qualitatively enhances the relaxational dynamics as compared to the case of normal diffusion. Parameters: $\rho_{0}=1, D_{\chi}=0, L=300$ (a,b), $L=400$ (c,d), $\beta(x)=\Theta(1-x)$, numerical time step $\tau=0.01$.

The emergence of long-range order in the case of superdiffusive transport can intuitively be understood by studying in which way macroscopic fluctuations decay. We quantify the temporal relaxation to the stationary state by preparing the system in the disordered state and, thereafter, observing how the system relaxes for zero noise $\left(D_{\chi}=0\right)$. After an initial transient, the system is locally ordered but globally disordered due to the emergence of free topological defects (vortices and antivortices) which eventually annihilate pairwise (see Fig. 3 for a visualization). In general, defects annihilate faster if the diffusivity of oscillators is high (cf. Fig. 4). However, the asymptotic behavior of the time dependent defect density $n(t)$ does not depend on the diffusion coefficient $\gamma$ for normal diffusion (black line in Fig. 4 4 ) $: n(t) \sim \ln t / t$ similar to the classical XY model [51]. On the other hand, the annihilation process is qualitatively accelerated towards a power-law like decay of the defect density $n(t) \sim t^{-\delta}$ with $\delta>1$ in the long-time limit, if particles perform Lévy flights indicating that superdiffusion of individual entities induces a novel regime of the relaxation process.

\section{FIELD THEORY}

We corroborate the simulation results by deriving and analyzing field equations for the relevant order parameters. The instantaneous particle density

$$
\rho(\mathbf{r}, t)=\sum_{j=1}^{N} \delta\left(\mathbf{r}-\mathbf{r}_{j}(t)\right)
$$

is a central quantity of interest, since the presence of density fluctuations distinguishes the nonequilibrium temporal dynamics of motile oscillators from lattice systems in 
equilibrium - the dynamic network of motile particles induces a fluctuating number of neighbors thus providing a source of nonthermal noise. In the stationary state, the mean density is constant since there are no forces acting among the particles thus excluding the emergence of density instabilities. Fluctuations around the mean are determined by the propagator $G_{\alpha}(\mathbf{r}, t)$ which also reflects the transition probability for a spatial displacement of a particle within a certain time interval:

$$
\begin{aligned}
& \langle\rho(\mathbf{r}, t)\rangle=\rho_{0} \\
& \left\langle\rho(\mathbf{r}, t) \rho\left(\mathbf{r}^{\prime}, t^{\prime}\right)\right\rangle-\rho_{0}^{2}=\rho_{0}\left[G_{\alpha}\left(\mathbf{r}-\mathbf{r}^{\prime}, t-t^{\prime}\right)-L^{-d}\right] .
\end{aligned}
$$

Correlations of the density field are proportional to the mean density and, therefore, the variance of the rescaled density $\delta \tilde{\rho}=\left[\rho / \rho_{0}-1\right]$ decreases proportional to $\rho_{0}^{-1}$ in accordance with the central limit theorem [52.

Along with the density describing the spatial distribution of particles, we introduce an order parameter field

$$
\mathbf{S}(\mathbf{r}, t)=|\mathbf{S}|\left(\begin{array}{c}
\cos \psi \\
\sin \psi
\end{array}\right)=\sum_{j=1}^{N}\left(\begin{array}{c}
\cos \chi_{j}(t) \\
\sin \chi_{j}(t)
\end{array}\right) \delta\left(\mathbf{r}-\mathbf{r}_{j}(t)\right)
$$

reflecting the local degree of synchronization. This field variable is related to the global order parameter $\Phi$ that was used to characterize particle based simulations via

$$
\Phi=\left\langle\left|\frac{1}{N} \int d^{d} r \mathbf{S}(\mathbf{r}, t)\right|^{2}\right\rangle=\frac{1}{N^{2}} \int d^{d} r d^{d} r^{\prime}\left\langle\mathbf{S}(\mathbf{r}, t) \cdot \mathbf{S}\left(\mathbf{r}^{\prime}, t\right)\right\rangle .
$$

Hence, an increase of the global order parameter - as observed in numerical simulations - is related to a qualitative change of the correlation function of $\mathbf{S}$ : correlations become long-ranged at the critical point.

The definition of the order parameter, Eq. (4), constitutes a change of variables from $\left\{\mathbf{r}_{j}(t), \chi_{j}(t)\right\}$ to the field $\mathbf{S}(\mathbf{r}, t)$. Therefore, the dynamics is obtained by application of stochastic calculus [48, 53. It turns out that the dynamics is coupled to the density and the nematic tensor $\mathcal{Q}=\sum_{j=1}^{N} \mathcal{M}\left[\chi_{j}(t)\right] \delta\left(\mathbf{r}-\mathbf{r}_{j}(t)\right)$, where

$$
\mathcal{M}[\varphi]=\left(\begin{array}{rr}
\cos (2 \varphi) & \sin (2 \varphi) \\
\sin (2 \varphi) & -\cos (2 \varphi)
\end{array}\right)
$$

Here, we use the closure relation $\mathcal{Q} \approx|\mathbf{S}|^{2} M[\psi] / \rho$ to obtain a self-consistent field equation for $\mathbf{S}(\mathbf{r}, t)$ :

$$
\begin{aligned}
\partial_{t} \mathbf{S}(\mathbf{r}, t) \approx & \gamma \Delta^{\alpha / 2} \mathbf{S}+\tilde{\beta} \Delta \mathbf{S}+\left[D_{\chi_{c}}^{(l)}-D_{\chi}-\epsilon|\mathbf{S} / \rho|^{2}\right] \mathbf{S} \\
& +\sqrt{D_{\chi} \rho(\mathbf{r}, t) / 2} \boldsymbol{\Pi} \cdot \boldsymbol{\eta}(\mathbf{r}, t)
\end{aligned}
$$

This field theory reflects the microscopic dynamics: the (fractional) Laplacian multiplied by the diffusivity $\gamma$ describes the transport of particles [41, 44] giving rise to (non-)local coupling; the coefficient $\tilde{\beta}=$ $\left[\int d^{d} r \beta(|\mathbf{r}|)|\mathbf{r}|^{2}\right] /\left[4 d \int d^{d} r^{\prime} \beta\left(\left|\mathbf{r}^{\prime}\right|\right)\right]$ stems from the interaction among the oscillators; the term in brackets indicates the emergence of local order via a pitchfork bifurcation for $D_{\chi}<D_{\chi_{c}}^{(l)}=1 / 2$, where $\epsilon=1 / 2$. Fluctuations on the macroscale are reflected by the Gaussian field $\boldsymbol{\eta}(\mathbf{r}, t)$ in Eq. (6), which is projected by the matrix

$$
\boldsymbol{\Pi}=\sqrt{1+\sqrt{1-|\mathbf{S} / \rho|^{4}}} \mathbb{1}-\sqrt{1-\sqrt{1-|\mathbf{S} / \rho|^{4}}} \mathcal{M}(\psi) .
$$

We emphasize (i) the multiplicative noise comprising the square root of the density and (ii) the non-Hamiltonian dynamics. Consequently, the probability to find a certain field configuration is generally not determined by a Boltzmann distribution, in turn reflecting the absence of temperature and the nonequilibrium nature of the model. In this regard, the dynamics of the system close to the transition point may potentially contain novel physics including a set of non-classical critical exponents in the low density regime. These questions which go beyond the scope of this work may be addressed in future studies. Here, we focus on the properties of the synchronized phase as well as the emergent dynamics of topological defects.

In the high density regime, fluctuations of the density can approximately be neglected according to Eq. (3) enabling us to link the nonequilibrium system featuring a dynamic network of short-ranged interactions to the field theory of the static XY model [54 57]. This nontrivial mapping, which connects two fundamentally different physical regimes, explains the phenomenology observed in simulations in several aspects. We deduce from Eq. (6) that the diffusivity $\gamma$ rescales the coupling coefficient $\beta$ in the case of Brownian diffusion $(\alpha=2)$. The structure of Eq. (6), however, does not change for $\gamma \rightarrow 0$ in that case. Therefore, the qualitative phenomenology of the XY model is observed if oscillators perform Brownian motion including a BKT transition towards QLRO whereas the equation is structurally different for superdiffusive motion.

Close to the critical point (in the disordered phase), the local order parameter is small, $|\mathbf{S} / \rho| \ll 1$, and therefore $\boldsymbol{\Pi} \approx 2 \cdot \mathbb{1}$ to lowest order. In this limit, the field equation (6) reduces to the Ginzburg-Landau model with additive white noise enabling us to determine the qualitative dependence of the transition point $D_{\chi_{c}}$ on model parameters [58, 59]:

$$
D_{\chi_{c}}=D_{\chi_{c}}^{(l)}\left[1-\frac{2 \epsilon}{\rho_{0}} \int^{\Lambda} \frac{d^{d} q}{(2 \pi)^{d}} \frac{1}{\gamma|\mathbf{q}|^{\alpha}+\tilde{\beta}|\mathbf{q}|^{2}}+\mathcal{O}\left(\epsilon^{2}\right)\right] .
$$

The integral in Eq. (7) runs over all wavevectors with $|\mathbf{q}|<\Lambda$, where $\Lambda$ is a cutoff parameter inversely proportional to the coarse-graining scale. Since the integrand is positive, the critical noise $D_{\chi_{c}}$ is shifted towards smaller noise values with respect to $D_{\chi_{c}}^{(l)}$ as observed in simulations (cf. Fig. 1). Only if the mobility or the particle density is high, the noise-induced shift of $D_{\chi_{c}}$ vanishes 
and the order-disorder transitions on the local and global level occur simultaneously: $D_{\chi_{c}} \simeq D_{\chi_{c}}^{(l)}$.

We focus now on the properties of locally ordered states, where $\mathbf{S}(\mathbf{r}, t) \approx S_{0}(\cos \psi(\mathbf{r}, t), \sin \psi(\mathbf{r}, t))$. In this regime, all quantities are determined by the phase field $\psi(\mathbf{r}, t)$, such as the correlation function $\left\langle\mathbf{S}(\mathbf{r}, t) \cdot \mathbf{S}\left(\mathbf{r}^{\prime}, t\right)\right\rangle \approx S_{0}^{2}\left\langle\cos \left[\psi(\mathbf{r}, t)-\psi\left(\mathbf{r}^{\prime}, t\right)\right]\right\rangle$. The dynamics of $\psi(\mathbf{r}, t)$ follows from Eq. (6):

$$
\partial_{t} \psi \approx \gamma \mathcal{C} \int d^{d} r^{\prime} \frac{\sin \left[\psi\left(\mathbf{r}^{\prime}, t\right)-\psi(\mathbf{r}, t)\right]}{\left|\mathbf{r}^{\prime}-\mathbf{r}\right|^{\alpha+d}}+\tilde{\beta} \Delta \psi+\sqrt{\varkappa} \varsigma .
$$

The noise strength is denoted by $\varkappa=D_{\chi} \rho_{0}(1+$ $\left.S_{0}^{2} / \rho_{0}^{2}\right) / S_{0}^{2}, \quad \mathcal{C}$ is a constant [60] and $\varsigma(\mathbf{r}, t)$ is a Gaussian white field [61. We assume further that $\psi(\mathbf{r}, t)$ varies slowly such that we can approximate $\sin \left[\psi\left(\mathbf{r}^{\prime}, t\right)-\psi(\mathbf{r}, t)\right] \approx\left[\psi\left(\mathbf{r}^{\prime}, t\right)-\psi(\mathbf{r}, t)\right]$ to obtain

$$
\partial_{t} \psi(\mathbf{r}, t) \approx \gamma \Delta^{\alpha / 2} \psi+\tilde{\beta} \Delta \psi+\sqrt{\varkappa} \varsigma(\mathbf{r}, t) .
$$

The solution of this linear equation is straightforward via Fourier transformation. One finds that the stationary phase field $\psi(\mathbf{r}, t)$ is Gaussian allowing us to calculate the order parameter correlations by the identity [57.

$$
\left\langle\mathbf{S}(\mathbf{r}, t) \cdot \mathbf{S}\left(\mathbf{r}^{\prime}, t\right)\right\rangle \approx S_{0}^{2} \exp \left[-\frac{1}{2}\left\langle\left[\psi(\mathbf{r}, t)-\psi\left(\mathbf{r}^{\prime}, t\right)\right]^{2}\right\rangle\right] .
$$

Accordingly, the order parameter correlation function is determined by the mean squared phase difference which follows from the solution of Eq. 8):

$$
\left\langle\left[\psi(\mathbf{r}, t)-\psi\left(\mathbf{r}^{\prime}, t\right)\right]^{2}\right\rangle=\varkappa \int^{\Lambda} \frac{d^{d} q}{(2 \pi)^{d}} \frac{1-e^{i \mathbf{q} \cdot\left(\mathbf{r}-\mathbf{r}^{\prime}\right)}}{\gamma|\mathbf{q}|^{\alpha}+\tilde{\beta}|\mathbf{q}|^{2}} .
$$

The mean squared phase difference at two points $\mathbf{r}$ and $\mathbf{r}^{\prime}$ behaves for large separations as $\left|\mathbf{r}-\mathbf{r}^{\prime}\right|^{\alpha-d}$, i.e. it decays for $\alpha<d$ indicating a long-ranged ordered state due to superdiffusive motion of particles. Note that the large scale dynamics essentially depends on the dimension of space. In contrast, microscopic details do not change the large scale properties qualitatively, thus indicating a form of universality.

Furthermore, the deterministic equation for $\psi(\mathbf{r}, t)$ supports vortex solutions $\psi(\mathbf{r}, t)= \pm \arg (\mathbf{r})+\psi_{0}$ in two dimensions as observed in numerical simulations (cf. Fig. 3, 4). We exploit Eq. (8) to determine the relaxational dynamics of the defect density from a qualitative scaling argument. By successive rescaling of space $\mathbf{r} \rightarrow b \mathbf{r}$ and time $t \rightarrow b^{\alpha} t$ with a positive scale parameter $b$, the diffusive coupling $\left(\tilde{\beta} \rightarrow b^{\alpha-2} \tilde{\beta}\right)$ as well as the noise term $\left(\varkappa \rightarrow b^{\alpha-2} \varkappa\right)$ are rendered irrelevant for $\alpha<2$ whereas $\gamma$ remains unchanged. Thus, the phase equation becomes scale invariant in the long time limit. Hence, the mean defect number in a cube of volume $L^{2}$ at time $t$ is asymptotically equal to the defect number in the domain of size $(b L)^{2}$ at time $b^{\alpha} t$ (cf. Fig. 5 for an illustration). Consequently, the defect density $n(t)$ is asymptotically a homogeneous function decaying as $n(t) \propto t^{-2 / \alpha}$, where the exponent explicitly depends on the motion type via $\alpha$, but is

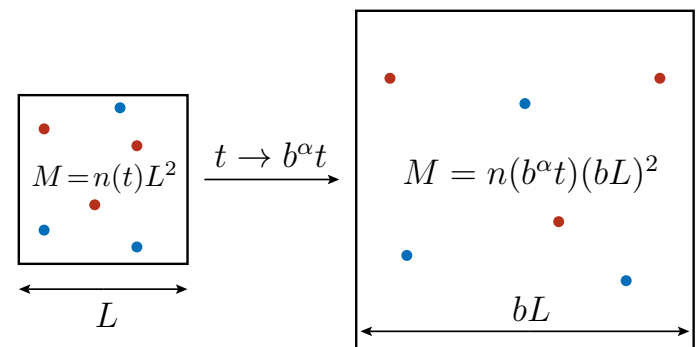

FIG. 5. Illustration of the self-similarity argument determining the decay of the density of topological defects. The dynamics of the phase field $\psi(\mathbf{r}, t)$, cf. Eq. (8), is asymptotically scale invariant under the rescaling $\mathbf{r} \rightarrow b \mathbf{r}$ and $t \rightarrow b^{\alpha} t$. Therefore, the mean number of defects $M$ in a cube of size $L^{2}$ at time $t$ (left panel) and in a cube $(b L)^{2}$ at time $b^{\alpha} t$ (right panel) is approximately equal, as illustrated above.

independent of microscopic details. Accordingly, the relaxation is accelerated in the case of superdiffusive transport as observed in numerical simulations (cf. Fig. 4).

\section{SUMMARY \& OUTLOOK}

We studied an ensemble of random walkers carrying internal clocks which are synchronized by local interactions. The phenomenology of this paradigmatic nonequilibrium model, as observed in numerical simulations, is remarkably rich. Numerical observations were corroborated by a non-Hamiltonian field theory including multiplicative noise terms which was analytically derived from the microscopic particle dynamics. The field theory reveals that the qualitative phenomenology of the system is not determined by mixing rates or, equivalently, the diffusion coefficients but rather by the motion type of particles.

For Brownian diffusion of individual oscillators, we report on a Berezinskii-Kosterlitz-Thouless transition from incoherence to quasi long-range order accompanied by the emergence of topological defects implying power-law like system size dependencies of all synchronization properties. We argued that the nonequilibrium system with diffusive particle transport exhibits the same qualitative behavior as an equilibrium system where particles are held fixed on a lattice. This implies that long-range order cannot emerge for dimensions smaller or equal to two in the thermodynamic limit and, thus, extends the predictions of the Mermin-Wagner theorem [27] - valid for equilibrium systems - to a nonequilibrium system with diffusive particle transport.

Furthermore, we showed that superdiffusive transport may give rise to long-range order in two and even in one dimension depending on the characteristics of the motion of individual entities. The emergence of long-range order in two dimensions relies on the accelerated annihilation dynamics of topological defects as confirmed by 
numerical simulations in two dimensions.

In short, we characterized in this work the conditions required for the emergence of long-range order in nonequilibrium systems. We believe that the stochastic dynamics of moving oscillators provides an interesting playground to study the interplay of local interaction and particle transport in the context of nonequilibrium statistical mechanics from a theoretical point of view, involving the mixture of normal fluctuations (oscillator dynamics) and anomalous fluctuations (superdiffusive motion) as well as the emergence of order and dynamics of topological defects in this context. Besides, the results may serve as a reference point for the description of specific experiments such as those mentioned in the introduction, where modeling may require a higher level of complexity, e.g. the addition of time delay [38].

\section{ACKNOWLEDGEMENT}

RG and MB gratefully acknowledge the support by the German Research Foundation via Grant No. GRK 1558. FP acknowledges support by the Agence Nationale de la Recherche (ANR) via JCJC project "BactPhys".
[1] A. F. Taylor, M. R. Tinsley, F. Wang, Z. Huang, and K. Showalter, Science 323, 614 (2009).

[2] M. S. Paoletti, C. R. Nugent, and T. H. Solomon, Phys. Rev. Lett. 96, 124101 (2006).

[3] T. Danino, O. Mondragón-Palomino, L. Tsimring, and J. Hasty, Nature 463, 326 (2010).

[4] Y.-J. Jiang, B. L. Aerne, L. Smithers, C. Haddon, D. IshHorowicz, and J. Lewis, Nature 408, 475 (2000).

[5] K. Horikawa, K. Ishimatsu, E. Yoshimoto, S. Kondo, and H. Takeda, Nature 441, 719 (2006).

[6] A. C. Oates, L. G. Morelli, and S. Ares, Development 139, 625 (2012).

[7] T. Gregor, K. Fujimoto, N. Masaki, and S. Sawai, Science 328, 1021 (2010).

[8] O. Sliusarenko, J. Neu, D. R. Zusman, and G. Oster, P. Natl. Acad. Sci. U.S.A. 103, 1534 (2006).

[9] H. Zhang, Z. Vaksman, D. B. Litwin, P. Shi, H. B. Kaplan, and O. A. Igoshin, PLoS Comput. Biol. 8, e1002715 (2012).

[10] J. Starruß, F. Peruani, V. Jakovljevic, L. SøgaardAndersen, A. Deutsch, and M. Bär, Interface Focus 2, 774 (2012).

[11] S. Fürthauer and S. Ramaswamy, Phys. Rev. Lett. 111, 238102 (2013).

[12] M. Leoni and T. B. Liverpool, Phys. Rev. Lett. 112, 148104 (2014).

[13] A. De Masi, P. A. Ferrari, and J. L. Lebowitz, Phys. Rev. Lett. 55, 1947 (1985).

[14] K. Kawasaki, Phys. Rev. 145, 224 (1966).

[15] R. J. Glauber, J. Math. Phys. 4, 294 (1963).

[16] P. Romanczuk, M. Bär, W. Ebeling, B. Lindner, and L. Schimansky-Geier, Eur. Phys. J. - Spec. Top. 202, 1 (2012).

[17] M. C. Marchetti, J.-F. Joanny, S. Ramaswamy, T. B. Liverpool, J. Prost, M. Rao, and R. A. Simha, Rev. Mod. Phys. 85, 1143 (2013).

[18] A. M. Menzel, Phys. Rep. 554, 1 (2015).

[19] J. Toner and Y. Tu, Phys. Rev. E 58, 4828 (1998).

[20] T. Vicsek and A. Zafeiris, Phys. Rep. 517, 71 (2012).

[21] H. Hinrichsen, Adv. Phys. 49, 815 (2000).

[22] U. C. Täuber, M. Howard, and B. P. Vollmayr-Lee, J. Phys. A - Math. Gen. 38, R79 (2005).

[23] M. Frasca, A. Buscarino, A. Rizzo, L. Fortuna, and S. Boccaletti, Phys. Rev. E 74, 036110 (2006).
[24] F. Peruani and G. J. Sibona, Phys. Rev. Lett. 100, 168103 (2008).

[25] O. Hallatschek and D. S. Fisher, Proc. Natl. Acad. Sci. U.S.A. 111, E4911 (2014).

[26] P. C. Hohenberg and B. I. Halperin, Rev. Mod. Phys. 49, 435 (1977).

[27] N. D. Mermin and H. Wagner, Phys. Rev. Lett. 17, 1133 (1966).

[28] A. Pikovsky, M. Rosenblum, and J. Kurths, Synchronization. A universal concept in nonlinear sciences, no. 12 in Cambridge Nonlinear Science Series (Cambridge University Press, 2001).

[29] F. Peruani, E. M. Nicola, and L. G. Morelli, New J. Phys. 12, 093029 (2010).

[30] K. Uriu, S. Ares, A. C. Oates, and L. G. Morelli, Phys. Rev. E 87, 032911 (2013).

[31] P. M. Chaikin and T. C. Lubensky, Principles of Condensed Matter Physics (Cambridge University Press, 1995).

[32] M. Porfiri, D. J. Stilwell, E. M. Bollt, and J. D. Skufca, Physica D 224, 102 (2006).

[33] J. D. Skufca and E. M. Bollt, Math. Biosci. Eng. 1, 347 (2004).

[34] M. Frasca, A. Buscarino, A. Rizzo, L. Fortuna, and S. Boccaletti, Phys. Rev. Lett. 100, 044102 (2008).

[35] N. Fujiwara, J. Kurths, and A. Díaz-Guilera, Phys. Rev. E 83, 025101 (2011).

[36] J. Gómez-Gardeñes, V. Nicosia, R. Sinatra, and V. Latora, Phys. Rev. E 87, 032814 (2013).

[37] K. Uriu, Y. Morishita, and Y. Iwasa, Proc. Natl. Acad. Sci. U.S.A. 107, 4979 (2010).

[38] K. Uriu, S. Ares, A. C. Oates, and L. G. Morelli, Phys. Biol. 9, 036006 (2012).

[39] V. Berezinskii, Sov. Phys. JETP 32, 493 (1971).

[40] J. M. Kosterlitz and D. J. Thouless, J. Phys. C (Solid State) 6, 1181 (1973).

[41] R. Klages, G. Radons, and I. M. Sokolov, Anomalous transport: foundations and applications (John Wiley \& Sons, 2008).

[42] For $\alpha=2$, the generalized noise $\boldsymbol{\xi}_{i}(t)$ reduces to Gaussian white noise with zero mean, $\left\langle\boldsymbol{\xi}_{i}(t)\right\rangle=0$, and $\delta$ correlations $\left\langle\xi_{i, \mu}(t) \xi_{j, \nu}(t)\right\rangle=2 \gamma \delta_{i j} \delta_{\mu \nu} \delta\left(t-t^{\prime}\right)$.

[43] G. Samoradnitsky and M. S. Taqqu, Stable non-Gaussian random processes: stochastic models with infinite variance, vol. 1 (CRC Press, 1994). 
[44] R. Metzler and J. Klafter, Phys. Rep. 339, 1 (2000).

[45] Y. Kuramoto, Prog. Theor. Phys. Supp. 79, 223 (1984).

[46] S. Shinomoto and Y. Kuramoto, Prog. Theor. Phys. 75, 1319 (1986).

[47] J. A. Acebrón, L. L. Bonilla, C. J. Pérez Vicente, F. Ritort, and R. Spigler, Rev. Mod. Phys. 77, 137 (2005).

[48] C. Gardiner, Stochastic Methods: A Handbook for the Natural and Social Sciences, Springer Series in Synergetics (Springer, 2010).

[49] The order parameter of globally coupled Kuramoto oscillators can be obtained from the exact relation $\Phi=I_{1}\left(\Phi / D_{\chi}\right) / I_{0}\left(\Phi / D_{\chi}\right)$, where $I_{n}(x)$ denote modified Bessel functions of the first kind.

[50] J. Quintanilla, S. Torquato, and R. M. Ziff, J. Phys. A Math. Gen. 33, L399 (2000).

[51] A. J. Bray, A. J. Briant, and D. K. Jervis, Phys. Rev. Lett. 84, 1503 (2000).

[52] W. Feller, An Introduction to Probability Theory and Its Applications, vol. 2 (Wiley, 1970).

[53] D. S. Dean, J. Phys. A - Math. Gen. 29, L613 (1996).
[54] M. E. Fisher, S.-k. Ma, and B. G. Nickel, Phys. Rev. Lett. 29, 917 (1972).

[55] M. Suzuki, Y. Yamazaki, and G. Igarashi, Phys. Lett. A 42, 313 (1972).

[56] B. Bergersen and Z. Rácz, Phys. Rev. Lett. 67, 3047 (1991).

[57] J. Zinn-Justin, Quantum field theory and critical phenomena (Oxford University Press, 2002).

[58] M. Bellac, Quantum and Statistical Field Theory (Oxford University Press, 1991).

[59] U. C. Täuber, Critical dynamics: a field theory approach to equilibrium and non-equilibrium scaling behavior (Cambridge University Press, 2014).

[60] The prefactor $\mathcal{C}=-2^{\alpha} / \pi^{d / 2} \cdot \Gamma\left(\frac{\alpha+d}{2}\right) / \Gamma\left(-\frac{\alpha}{2}\right)$ stems from the definition of the fractional Laplacian $\Delta^{\alpha / 2}$ acting in Fourier space as a multiplication by $\left(-|\mathbf{q}|^{\alpha}\right)$, where $\mathbf{q}$ denotes the wavevector, cf. 41, 44.

[61] Besides Gaussianity, Gaussian white fields $\xi_{\mu}\left(x_{1}, x_{2}, \ldots, x_{n}\right)$ are unbiased, $\left\langle\xi_{\mu}\left(x_{1}, x_{2}, \ldots, x_{n}\right)\right\rangle=$ 0 , and $\delta$-correlated in all arguments: $\left\langle\xi_{\mu}\left(x_{1}, x_{2}, \ldots, x_{n}\right) \xi_{\nu}\left(x_{1}^{\prime}, x_{2}^{\prime}, \ldots, x_{n}^{\prime}\right)\right\rangle=\delta_{\mu \nu} \delta\left(\mathbf{x}-\mathbf{x}^{\prime}\right)$. 